\title{
AKT serine/threonine protein kinase modulates bufalin-triggered intrinsic pathway of apoptosis in CAL 27 human oral cancer cells
}

\author{
SHIH-CHANG TSAI ${ }^{1}$, CHI-CHENG LU ${ }^{8}$, CHAO-YING LEE $^{4}$, YUNG-CHANG LIN ${ }^{9}$, JING-GUNG CHUNG ${ }^{1}$, \\ SHENG-CHU KUO $^{7}$, SAKAE AMAGAYA $^{6,11}$, FEI-NA CHEN ${ }^{2}$, MICHAEL YUANCHIEN CHEN ${ }^{5,10}$, \\ SHIH-FENG CHAN ${ }^{1}$ and JAI-SING YANG ${ }^{3}$

\begin{abstract}
Departments of ${ }^{1}$ Biological Science and Technology, ${ }^{2}$ Social Medicine and ${ }^{3}$ Pharmacology, Schools of ${ }^{4}$ Pharmacy and ${ }^{5}$ Dentistry, ${ }^{6}$ Tsuzuki Institute for Traditional Medicine, ${ }^{7}$ Graduate Institute of Pharmaceutical Chemistry,

China Medical University, Taichung 404; Departments of ${ }^{8}$ Life Sciences and ${ }^{9}$ Veterinary Medicine, National Chung Hsing University, Taichung 402; ${ }^{10}$ Department of Oral Maxillofacial Surgery, China Medical University Hospital, Taichung 404, Taiwan, R.O.C.; ${ }^{11}$ Department of Kampo Pharmaceutical Sciences, Nihon Pharmaceutical University, Saitama 362-0806, Japan
\end{abstract}

Received May 4, 2012; Accepted July 6, 2012

DOI: 10.3892/ijo.2012.1605

\begin{abstract}
Bufalin has been reported to induce apoptosis in a variety of cancers but little is demonstrated in oral squamous cell carcinoma (OSCC) cells. The present study investigated the inhibition of proliferation, cell cycle arrest and apoptotic effects of bufalin in CAL 27 human oral cancer cells. Bufalin inhibited the growth of CAL 27 cells in a concentration-dependent manner and an $\mathrm{IC}_{50}$ value of bufalin was about $125 \mathrm{nM}$ for $24 \mathrm{~h}$ treatment using the MTT assay. Moreover, the cell cycle distribution was arrested at the $G_{0} / G_{1}$ phase in CAL 27 cells after bufalin exposure. Upon bufalin stimulation, the expression of Bcl-2 was significantly decreased while that of cytochrome $c$, Apaf- 1 and AIF was increased compared to the control group by western blot analysis. An increase in the expression of the active form of caspases was found in bufalin-treated cells, and the caspase activities were also elevated. Bufalin-triggered apoptosis was blocked by specific inhibitors of caspase-9 (z-LEHD-fmk) and caspase-3 (z-DEVD-fmk), respectively. In contrast, CAL 27 cells overexpressing constitutively active AKT (CAL 27/CA-AKT) were exposed to bufalin at different concentrations, and cell growth remained unchanged. Bufalin exhibited minimal apoptotic effects on CAL 27/CA-AKT cells. Taken together, bufalin induced $\mathrm{G}_{0} / \mathrm{G}_{1}$ phase arrest and provoked the intrinsic apoptotic pathway via AKT activation in CAL 27 cells. Our data suggest that bufalin could be potentially efficacious in the treatment of oral cancer in the future.
\end{abstract}

Correspondence to: Dr Jai-Sing Yang, Department of Pharmacology, China Medical University, 91, Hsueh-Shih Road, Taichung 40402, Taiwan, R.O.C.

E-mail: jaising@mail.cmu.edu.tw

Key words: bufalin, constitutively active, $\mathrm{G}_{0} / \mathrm{G}_{1}$ phase arrest, apoptosis, caspase, CAL 27 oral cancer cells, AKT

\section{Introduction}

Oral squamous cell carcinoma (OSCC) is the most common head and neck cancer which exhibits frequent lymph node metastasis and local invasion, causing poor prognosis $(1,2)$. The addiction to betel, tobacco, and alcohol is found to be highly correlated with the risk of HNSCC (3) and the studies in this area might lead to new approaches in the prevention and treatment of this important group of human cancers (4).

Apoptosis involves a cascade of molecular changes such as morphology changes, chromatin condensation, and DNA fragmentation $(5,6)$. Abnormal regulation of apoptosis leads to many human disorders including autoimmune disease and cancer. Thus, understanding the mechanisms of apoptosis is an important strategy for treatment of cancer (6,7). Several gene products have been demonstrated to be critical in the regulation of apoptosis $(8,9)$. For example, caspases are synthesized as proenzymes and become activated by cleavage. Caspase activation is often regulated by various cellular factors, including members of the $\mathrm{Bcl}-2$. The $\mathrm{Bcl}-2$ protein family is divided into two functional subfamilies: pro-apoptotic proteins (Bax and Bid) and anti-apoptotic proteins (Bcl-2 and Bcl-xL) $(8,10)$. The family members translocate to the mitochondria and mediate the membrane potential to induce cytochrome $c$ release. Cytosolic cytochrome $c$ is further involved in caspase activation. The caspase cascade is a key pathway in apoptotic signal transduction, and can be divided into two types of subfamilies: upstream initiator caspases (caspase-8 and -9) and downstream effector caspases (caspase-3, -6 and -7), which directly induce the final events of apoptosis $(6,11,12)$.

Bufalin, a cardioactive C-24 steroid, is the major component of the traditional Chinese medicine Chan-Su obtained from the skin and parotid venom glands of the toad (13-15). A previous study has shown that bufalin is applied for a treatment of heart failure and used for a variety of biological activities, such as blood pressure stimulation and antineoplastic activities (14). In addition, bufalin processed biological functions as inhibitors of 
$\mathrm{Na}^{+} / \mathrm{K}^{+}$-ATPase and topoisomerase II, leading to protein-linked DNA double-strand breaks $(14,16)$. It has been reported that the topoisomerase inhibitor, etoposide and adriamycin are widely prescribed anticancer drugs which inhibit cell proliferation and induce apoptosis in numerous cancer cell lines $(17,18)$. Our earlier study showed that bufalin is found to inhibit cell growth at $G_{0} / G_{1}$ phase of the cell cycle and to induce apoptosis in a dose-dependent manner, which was associated with both mitochondria-regulated and death receptor-initiated pathways (19). Furthermore, bufalin has been found to inhibit Bcl-2 and c-myc in human leukemia cells (20) and to induce apoptosis of human prostate cancer cells in part with Fas stimulation, cytochrome $c$ release and caspase activation (15). Additionally, bufalin has also been found to induce apoptosis by upregulating the expression of Bax (21) and suppressing orthotopic transplantation tumor in nude mice in human hepatocellular carcinoma cells (22). Therefore, bufalin is thought to be a valuable anticancer drug.

There is limited information, however, on the cellular and molecular mechanisms underlying the bufalin-induced apoptosis in human oral cancer cells. The purpose of this study was to define the biological and therapeutic effects of bufalintreated human oral cancer cells for the first time. This study was designed to: i) evaluate the cytotoxicity effects of bufalin; ii) characterize the effect of bufalin on the cell cycle; iii) investigate the apoptotic effects of bufalin by analyzing the protein expression in CAL 27 human oral cancer cells in vitro.

\section{Materials and methods}

Chemicals and reagent. Dulbecco's modified Eagle's medium (DMEM), L-glutamine, fetal bovine serum (FBS), penicillin/streptomycin and Trypsin-EDTA were purchased from Gibco/Life Technologies (Carlsbad, CA, USA). 4,6-diamidino2-phenylindole dihydrochloride (DAPI), dimethyl sulfoxide (DMSO), propidium iodide (PI), bufalin, Triton X-100 and anti- $\beta$-actin antibody were obtained from Sigma-Aldrich Corp. (St. Louis, MO, USA). Caspase-3 inhibitor (z-DEVD-fmk, Cat. 264155), caspase-9 inhibitor (z-LEHD-fmk, Cat. 218761), anti-p-AKT (Ser473) (Cat. 07-310), anti-AKT (Cat. 05-591) and Immobilon Western Chemiluminescent HRP substrate (Cat. WBKLS0500) were bought from Merck Millipore (Billerica, MA, USA). Tdt-mediated deoxyuridine triphosphate nick end labeling (TUNEL) assay kit (in situ Cell Death Detection Kit, Fluorescein) was purchased from Roche Diagnostics (Boehringer Mannheim, Mannheim, Germany). These primary antibodies (anti-cyclin D1, anti-p-BAD, anti-BAD, anti-Bcl-2, anti-cytochrome $c$, anti-Apaf-1 and anti-AIF) and horseradish peroxidase (HRP) conjugated second antibodies for western blot analysis were obtained from Santa Cruz Biotechnology Inc. (Santa Cruz, CA, USA). The primary antibodies (anti-caspase-9 and anti-caspase-3) were obtained from Cell Signaling Technology (Danvers, MA, USA).

Cell culture and bufalin treatment. CAL 27 (CRL-2095) cell line was purchased from the American Type Culture Collection (Manassas, VA, USA). Cells were grown in DMEM supplemented with $10 \% \mathrm{FBS}, 100 \mathrm{U} / \mathrm{ml}$ penicillin and $100 \mu \mathrm{g} / \mathrm{ml}$ streptomycin at $37^{\circ} \mathrm{C}$ in a humidified atmosphere of $5 \% \mathrm{CO}_{2}$ atmosphere incubator. Cells were treated with bufalin for indi- cated concentrations as in each experiment. Equivalent volume of $0.1 \%$ DMSO was used as vehicle control.

DNA construct and transfection. Constitutively active AKT was a gift from Dr Way (Department of Biological Science and Technology, China Medical University) and subcloned into pcDNA3 (Invitrogen/Life Technologies). CAL 27 cells were transfected with either an empty vector (pcDNA3), or a constitutively active AKT construct (pcDNA3-CA-AKT) using Arresti-In transfection reagent and performed according to the manufacturer's instructions (GenDiscovery Biotechnology, Taipei, Taiwan). Cells were selected in neomycin 48 post-transfection $(23,24)$. Viable stably transfected cells expressing constitutively active AKT were analyzed by western blotting as described elsewhere $(5,25)$.

Cell viability assay. The effects of bufalin on cell viability were determined using the 3-(4,5-dimethylthiazol-2-yl)-2,5-diphenyltetrazolium bromide (MTT, Sigma-Aldrich Corp.) assay. Briefly, $1 \times 10^{4}$ cells per well were seeded in 96-well culture plates. After overnight incubation, the cells were treated with different concentrations of bufalin $(0,50,100,150$ or $200 \mathrm{nM})$ for $24 \mathrm{~h}$. The cells were treated with $50 \mu \mathrm{l}$ of $5 \mathrm{mg} / \mathrm{ml}$ MTT for $4 \mathrm{~h}$ at $37^{\circ} \mathrm{C}$ and the resulting formazan crystals were dissolved in dimethyl sulfoxide (DMSO). The absorbance was measured by microplate spectrophotometer (Bio-Tek Instruments Inc., Winooski, VT, USA) at $570 \mathrm{~nm}$. Results were expressed as percentage of the controls, which were arbitrarily assigned $100 \%$ viability. Viability assays were performed in triplicate from three independent experiments. The $50 \%$ inhibitory concentration $\left(\mathrm{IC}_{50}\right)$ of bufalin was calculated as described previously $(26,27)$.

Determination for cell morphology. Cells ( $2 \times 10^{5}$ cells/well) were maintained in 24-well plates and then were treated with 0 , 50, 100 and $200 \mathrm{nM}$ of bufalin. Cell morphological examination was determined utilizing a phase-contrast microscope as previously described (19). Chromatin condensation was detected using the DAPI staining method as previously described $(19,28)$. CAL 27 cells were incubated with $100 \mathrm{nM}$ bufalin for $0,12,24$ and $48 \mathrm{~h}$. After that, cells were fixed gently by putting $70 \%$ ethanol, stained with DAPI, and then photographed using a fluorescence microscope.

Analysis for cell cycle progression by flow cytometry. Cells ( $2 \times 10^{5}$ cells/well) in 24-well plates were exposed to different concentrations of bufalin for $24 \mathrm{~h}$. For determination of cell cycle phase and apoptosis, cells were then collected, fixed in $70 \%$ ice-cold ethanol overnight, washed in PBS once, and resuspended in PBS containing $40 \mu \mathrm{g} / \mathrm{ml}$ PI, $0.1 \mathrm{mg} / \mathrm{ml}$ RNase A and $0.1 \%$ Triton X-100 in dark room for $30 \mathrm{~min}$. Cell cycle distribution and apoptotic nuclei were determined by flow cytometry (BD Biosciences, FACSCalibur flow cytometer, San Jose, CA, USA). The percentages of cells in $G_{1}, S$, and $G_{2} / M$ phases were analyzed using CellQuest Pro Software (BD Biosciences) as described previously $(29,30)$.

Assessment of apoptotic cells by TUNEL staining. TUNEL staining was performed according to the manufacturer's instructions (Roche Diagnostics). Cells $\left(2 \times 10^{5}\right.$ cells $\left./ \mathrm{ml}\right)$ in 24 -well plates were treated without or with $125 \mathrm{nM}$ bufalin for $24 \mathrm{~h}$. 
Cells were harvested and immediately incubated with terminal deoxynucleotidyl transferase (TdT) enzyme at $37^{\circ} \mathrm{C}$ for $1 \mathrm{~h}$. Following TUNEL staining, all samples were washed once with PBS and resuspended in $0.5 \mathrm{ml}$ of PBS containing PI $(10 \mu \mathrm{g} / \mathrm{ml})$ and DNase free-RNase A $(200 \mu \mathrm{g} / \mathrm{ml})$. TUNEL positive cells were analyzed by flow cytometry. The fluorescence intensity was quantified by BD Pro CellQuest software. TUNEL assays were performed in triplicate from three independent experiments as described previously (19,27).

Assay for caspase-3 activity. Caspase-3 colorimetric assay kit (R\&D Systems, Inc., Minneapolis, MN, USA) was aped according to the manufacturer's recommendations. In brief, cells $\left(1 \times 10^{7}\right.$ cells/flask) in T75 flasks were incubated with $125 \mathrm{nM}$ bufalin for $24 \mathrm{~h}$. Cells were harvested and lysed in a cold Lysis buffer (provided in the kit). Cell lysates (50 $\mu \mathrm{g}$ protein) were incubated with caspase-3 specific substrate (Ac-IETD-pNA) for $1 \mathrm{~h}$ at $37^{\circ} \mathrm{C}$. The caspase activity was determined by measuring cleavage of chromogenic caspase substrates (pNA) at $\mathrm{OD}_{405}$ in a microplate spectrophotometer (BioTek Instruments Inc.) $(27,31)$.

Western blot analysis. Cells $\left(1 \times 10^{7}\right.$ cells/flask) were seeded in T75 flasks and incubated with or without $125 \mathrm{nM}$ bufalin for $24 \mathrm{~h}$. Total proteins were prepared and determined as previously described $(25,28)$. Briefly, the protein concentration was measured using a BCA assay kit (Pierce Chemical, Rockford, IL, USA). Equal amounts $(40 \mu \mathrm{g})$ of proteins were boiled for $5 \mathrm{~min}$, separated by $12 \%$ SDS-PAGE, and then electro-transferred to Immobilon-P transfer membrane PVDF. The transferred membranes were blocked for $1 \mathrm{~h}$ with Tris-buffered saline/Tween-20 containing 5\% non-fat dry milk and incubated with primary antibodies at $4^{\circ} \mathrm{C}$ overnight. Membranes were washed three times with Tris-buffered saline/Tween-20 for $10 \mathrm{~min}$ and incubated with secondary HRP-conjugated antibody $(5,25)$. Signals of the blots were detected using an enhanced cheniluminescence (ECL) kit and developed in Kodak Bio-MAX MR film (Eastman Kodak, Rochester, NY, USA). The band density was quantified using National Institute of Health (NIH) ImageJ 1.45 program (28). Blots were probed with $\beta$-actin antibody used as the loading control.

Statistical analysis. All the statistical results were expressed as the mean \pm SD of triplicate samples. Statistical analyses of data were done using one-way ANOVA followed by Student's t-test, and $\mathrm{p}<0.05$ was considered significant.

\section{Results}

Effects of bufalin on the proliferation and viability of CAL 27 cells. Firstly, we investigated the effects of bufalin treatment on the growth of CAL 27 cells. As shown in Fig. 1, bufalin significantly reduced the cell viabilities of CAL 27 cells after $24 \mathrm{~h}$ exposure in a concentration-dependent manner. $\mathrm{IC}_{50}$ values were calculated to be about $125 \mathrm{nM}$ for $24 \mathrm{~h}$. Thus, $125 \mathrm{nM}$ was applied for all subsequent experiments. To determine whether the observed decrease in cell viability is associated with apoptosis, we examined the nuclear morphology under a phasecontrast microscope. In the control cells no significant changes

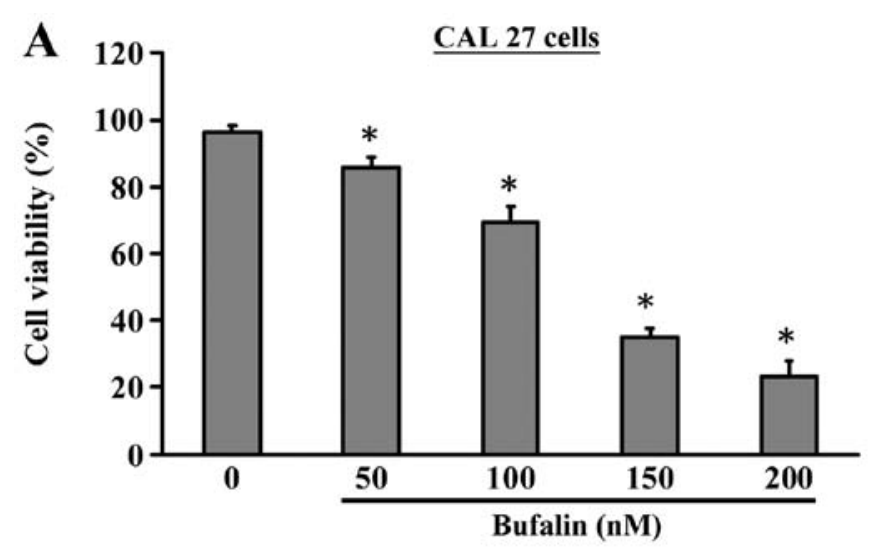

B

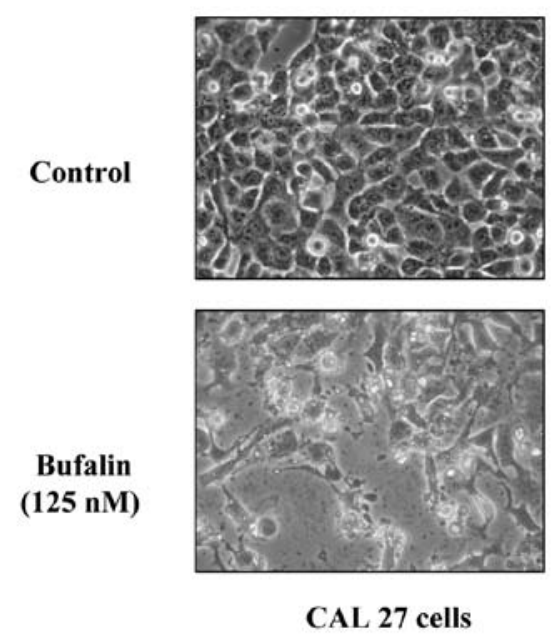

Figure 1 . The viability suppression effects of bufalin treatment on CAL 27 cells Cells were seeded in 96-well plates and treated with various concentrations of bufalin for $24 \mathrm{~h}$. After the end of treatment, cell viability was determined by MTT assay. The values were calculated relative to the control group $(0 \mu \mathrm{M})$. The results are mean \pm SD and representative of three independent experiments. ${ }^{*} \mathrm{p}<0.05$ versus the control group. (B) Representative microphotographs were taken after CAL 27 cells were treated with $125 \mathrm{nM}$ of bufalin for $24 \mathrm{~h}$ with a 200x magnification factor and showed apoptotic morphological changes. Similar results were obtained from three independent experiments.

were seen in cell nuclei or cell membrane integrity, whereas CAL 27 cells treated with $125 \mathrm{nM}$ of bufalin for $24 \mathrm{~h}$ showed various extent of cell shrinkage, volume reduction, apoptotic body formation and cell blebbing (Fig. 1B). These results suggest that bufalin exhibited a significant apoptosis-inducing effect on CAL 27 cells.

Cell cycle analysis of CAL 27 cells after exposure to bufalin. To elucidate whether growth inhibition by bufalin is associated with apoptosis, we determined apoptotic features by measurement of the amount of cells in the sub- $G_{1}$ phase in CAL 27 cells in vitro. The results from flow cytometric assay using PI staining revealed that treatment with bufalin resulted in increased accumulation of $\mathrm{G}_{0} / \mathrm{G}_{1}$ phase in CAL 27 cells and this effect is dose-dependent (Fig. 2A and B). Also, we found that bufalin increased sub- $\mathrm{G}_{1}$ population (apoptosis) in CAL 27 cells (Fig. 2A). These results suggest that bufalin inhibited proliferation of CAL 27 cells through $G_{0} / G_{1}$ phase arrest and induction of apoptotic cell death. 
A

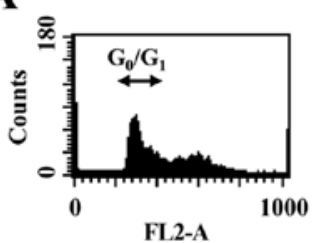

Control

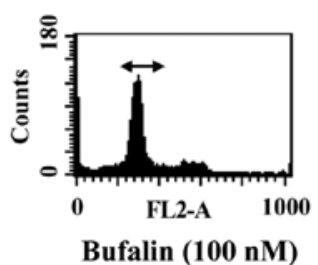

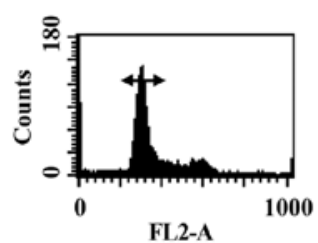

Bufalin (50 nM)

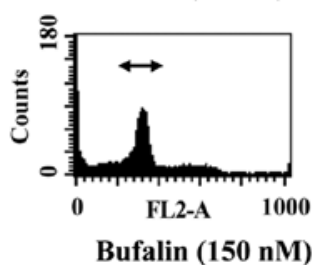

B

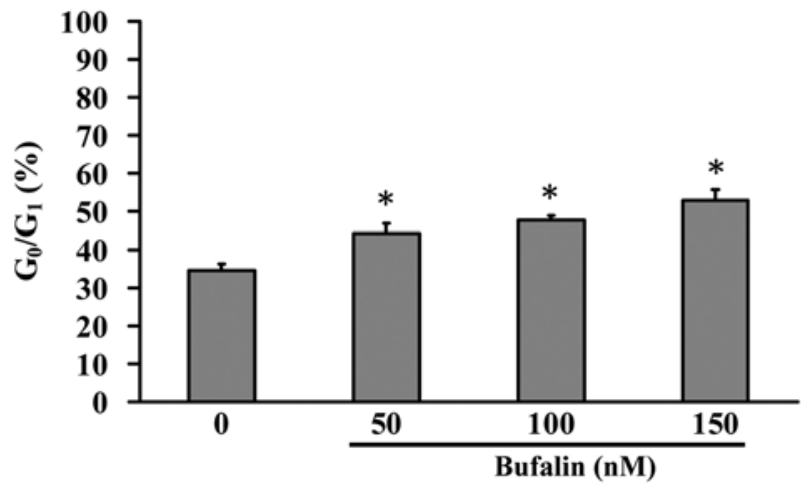

Figure 2. Bufalin induces concentration-dependent $\mathrm{G}_{0} / \mathrm{G}_{1}$ phase arrest in CAL 27 cells. (A) Cells were treated with bufalin at different concentrations $(0,50,100$ and $150 \mathrm{nM}$ ) for $24 \mathrm{~h}$. Cell cycle distribution was measured by flow cytometry as described in Materials and methods. The data are representative of three independent experiments. (B) The results represent the percentage of $\mathrm{G}_{0} / \mathrm{G}_{1}$ cells and mean \pm SD of three independent experiments. " $\mathrm{p}<0.05$ versus the control group.

A

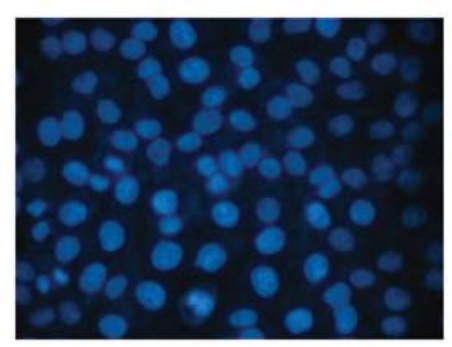

Control

B

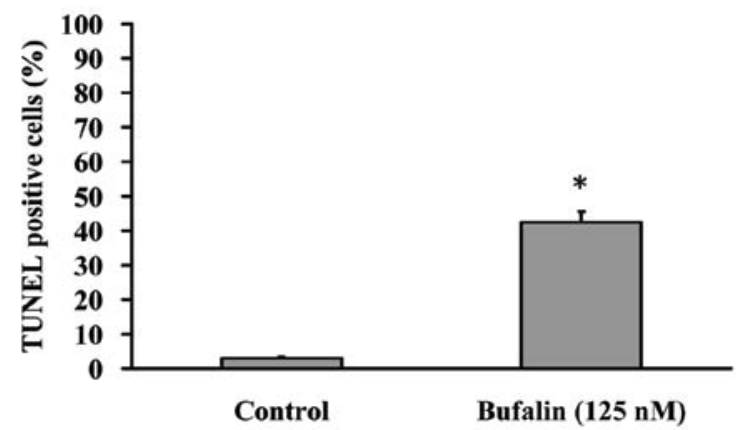

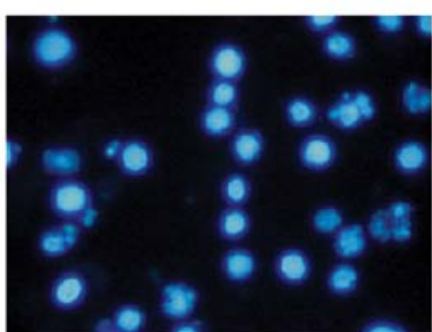

Bufalin (125 nM)

C

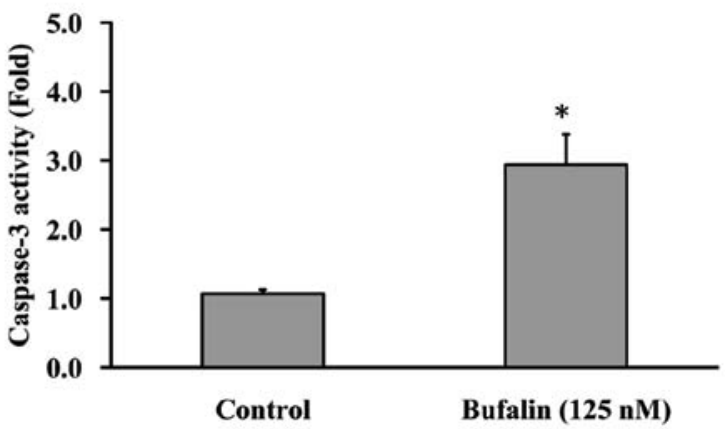

Figure 3. Bufalin-induced apoptosis is mediated by activation of caspase-3 in CAL 27 cells. Cells were treated with $125 \mathrm{nM}$ bufalin for $24 \mathrm{~h}$ and (A) subsequently stained with DAPI to determine chromatin condensation. Data are representative of three independent experiments with similar results. (B) After treatment, apoptotic cells were measured by TUNEL assay. (C) Effect of bufalin on caspase-3 activity in CAL 27 cells. The results are mean \pm SD and representative of three independent experiments. " $\mathrm{p}<0.05$ versus the control group.

Apoptotic feathers in bufalin-treated CAL 27 cells. Cells were treated with $125 \mathrm{nM}$ bufalin for $24 \mathrm{~h}$, then stained with DAPI and analyzed under a fluorescence microscope (Fig. 3A). In addition, the average percentage of apoptotic cells (TUNEL positive cells) increased from $5 \%$ of the control to $40 \%$ by DAPI/TUNEL double staining (Fig. 3B). In the present study, we investigated the possible mechanisms of bufalin-induced apoptosis in CAL 27 cells. Since caspases are known to play a pivotal role in mediating various apoptotic signaling $(8,10)$, we measured the activity of effector caspase (caspase-3) in bufalin-treated cells. Fig. 3C shows that exposure of CAL 27 cells to $125 \mathrm{nM}$ of bufalin led to increased levels of activated caspase-3. Taken together, we concluded that $125 \mathrm{nM}$ bufalin decreased the percentage of viable CAL 27 cells through apoptotic cell death. Moreover, caspases are central regulators of the apoptotic pathway.

Bufalin inhibits the growth of CAL 27 cells via the AKT signaling pathway. We investigated the effects of bufalin treat- 
A
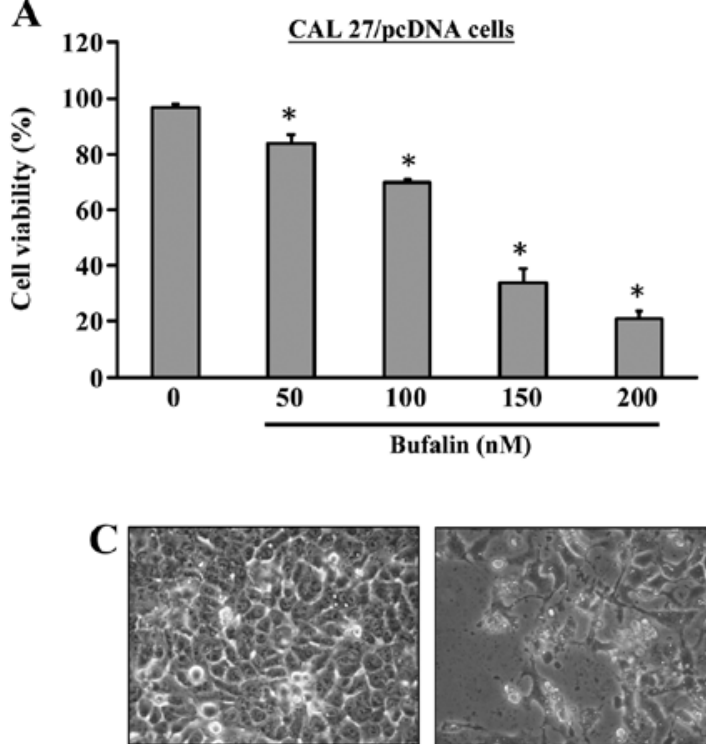

Control

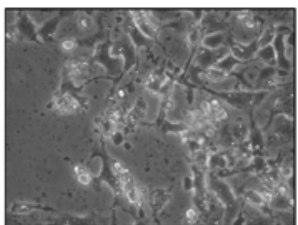

Bufalin (125 nM)

CAL 27/pcDNA cells
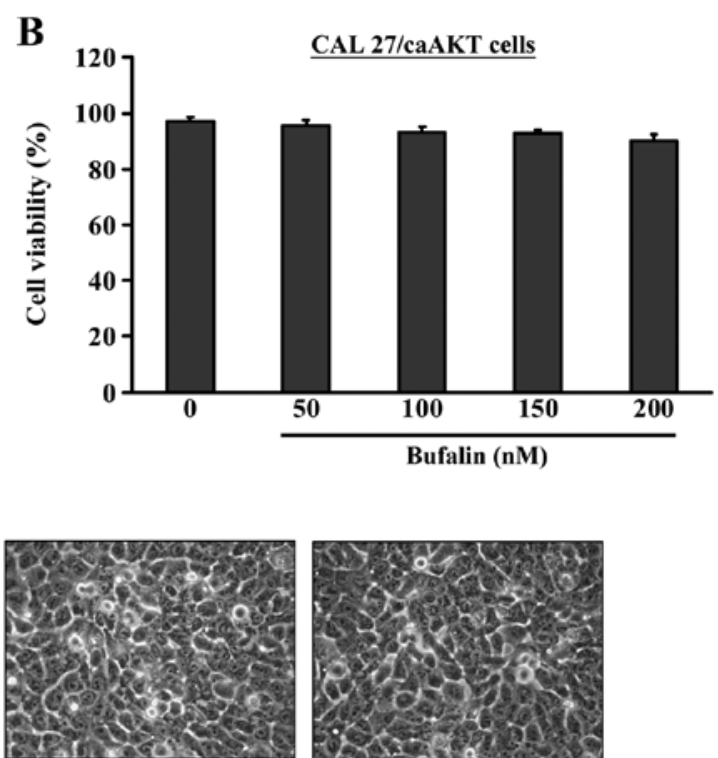

Control

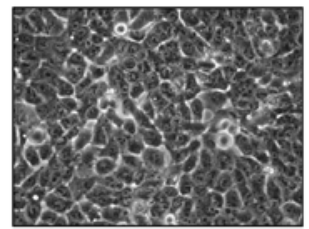

Bufalin (125 nM)

CAL 27/caAKT cells

Figure 4. The effects of bufalin treatment on CAL 27/pcDNA or CAL 27/CA-AKT cells. (A) CAL 27/pcDNA cells were seeded in 96-well plates and treated with various concentrations of bufalin for $24 \mathrm{~h}$. After the end of treatment, cell viability was determined by MTT assay. The values were calculated relative to the control group $(0 \mu \mathrm{M})$. (B) CAL 27/CA-AKT cells were treated as described in Materials and methods. Data are mean \pm SD and representative of three independent experiments. ${ }^{*}$ p $<0.05$ versus the control group ( $0 \mu \mathrm{M}$ bufalin). (C) Morphology changes were observed under a microscope. Data are representative of three independent experiments with similar results.

ment on the growth of CAL 27/pcDNA or CAL 27/CA-AKT cells. Results in Fig. 4A indicated that CAL 27/pcDNA cells were inhibited in growth and viability was reduced by bufalin in a concentration-dependent manner. To further determine if the observed decrease in cell viability is associated with apoptosis, we investigated the nuclear morphological changes CAL 27/pcDNA cells. Fig. 4C demonstrates that the control cells were not significantly changed in cell nuclei and cell membrane integrity, whereas bufalin-treated CAL 27/pcDNA cells showed various extent of chromatin condensation, nuclear fragmentation and destruction of cell membrane integrity after a 24-h incubation particularly with $125 \mathrm{nM}$ bufalin. Typical apoptotic nuclei were observed as early as $24 \mathrm{~h}$ in CAL 27/ pcDNA cells after treatment with bufalin. Characteristically morphological changes of apoptosis were also observed under a microscope, including cell shrinkage, volume reduction, chromatin condensation, cell blebbing and formation of membrane embedded apoptotic bodies (Fig. 4C). Strikingly, bufalin had minimal apoptotic effects (alteration of cell viability) on CAL 27/CA-AKT cells (Fig. 4B and C).

Bufalin causes cell cycle arrest at $G_{0} / G_{1}$ phase in CAL 27/ pcDNA but not in CAL 27/CA-AKT cells. To elucidate whether growth inhibition by bufalin is associated with apoptosis via the AKT signaling pathway, we explored the amount of cells in the $\mathrm{G}_{0} / \mathrm{G}_{1}$ phase in CAL 27/pcDNA and CAL 27/CA-AKT cells. The results from flow cytometric assay using PI staining revealed that treatment with $50-150 \mathrm{nM}$ bufalin resulted in increased accumulation of $\mathrm{G}_{0} / \mathrm{G}_{1}$ phase in CAL 27/pcDNA cells and this effect is dose-dependent (Fig. 5A and B). We also observed the sub- $\mathrm{G}_{1}$ population in CAL 27/pcDNA cells (Fig. 5A). In contrast, bufalin did not significantly affect the cell cycle arrest and had minimal apoptotic effects on CAL 27/ CA-AKT (Fig. 5A and B). Based on these observations, we propose that bufalin-induced $\mathrm{G}_{0} / \mathrm{G}_{1}$ phase arrest and apoptotic death in CAL 27 cells is carried out through the AKT signaling pathway.

Bufalin triggers apoptosis in CAL 27/pcDNA but not in CAL 27/CA-AKT cells. To confirm whether AKT expressionmodulated bufalin-induced apoptosis in CAL 27 cells, CAL 27/pcDNA and CAL 27/CA-AKT cells were used to investigate DAPI staining, TUNEL assay and caspase-3 activity. Cells were treated with $125 \mathrm{nM}$ bufalin for $24 \mathrm{~h}$ and then stained with DAPI. Data in Fig. 6A show that bufalin stimulated chromatin condensation in CAL 27/pcDNA but no effect was found in CAL 27/pcDNA cells. In addition, the average percentage of apoptotic cells (TUNEL positive cells) increased from 5\% of the control to $40 \%$ (Fig. 6B). In the present study, we investigated the possible mechanisms of bufalin-induced apoptosis in CAL 27 cells and found that AKT signaling might be involved in this event. Caspase-3 is known to play a pivotal role in mediating various apoptotic signaling $(8,10)$, and then we measured the activity of effector caspase- 3 in bufalin-treated cells. As illustrated in Fig. 6C, exposure of CAL 27 cells to $125 \mathrm{nM}$ bufalin led to increased caspase-3 activity, but no significant effect occurred in CAL 27/CA-AKT cells after bufalin incubation.

Effects of caspase-9 and caspase-3 inhibitors on bufalininduced apoptosis in CAL 27, CAL 27/pcDNA and $C A L$ 27/CA-AKT cells. To further investigate the involvement of intrinsic caspase signals in bufalin-induced apoptosis, both of z-DEVD-fmk (a caspase-9 inhibitor) and z-LEHD-fmk (a 
A

CAL 27/peDNA cells
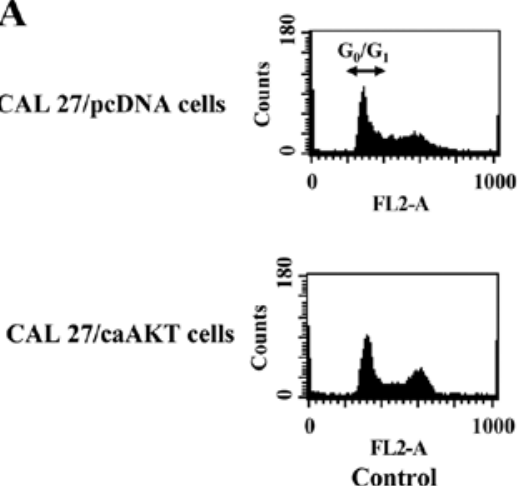
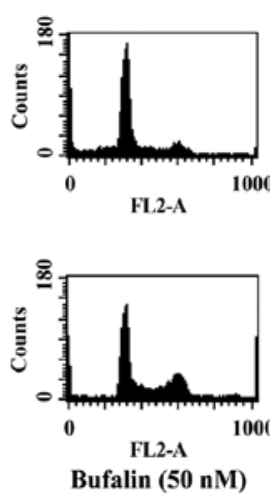
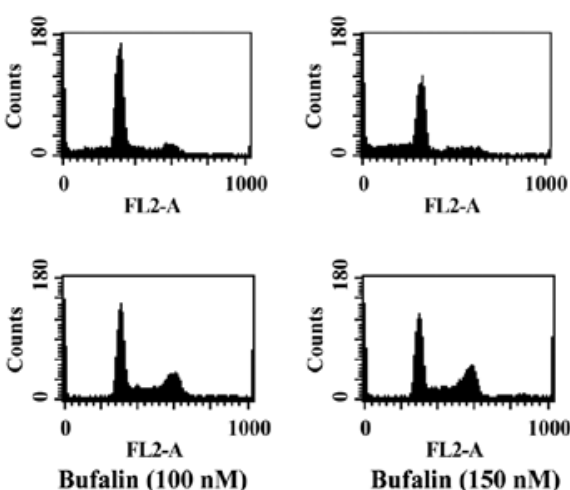

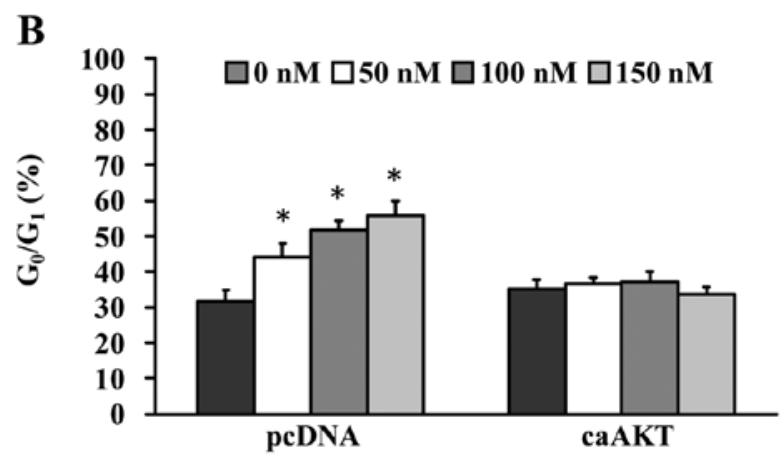

Figure 5. Bufalin induces concentration-dependent cell cycle arrest in CAL 27/pcDNA or CAL 27/CA-AKT cells. Cells were treated with bufalin at different concentrations $(0,50,100$ and $150 \mathrm{nM})$ for $24 \mathrm{~h}$. (A) Respective profiles from flow cytometry and (B) the results are mean \pm SD and representative of three independent experiments. ${ }^{\mathrm{p}}<0.05$ versus the control group $(0 \mathrm{nM})$.

$\mathbf{A}$

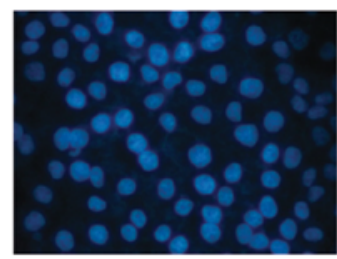

Control

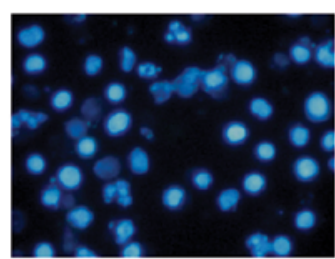

Bufalin (125 nM)

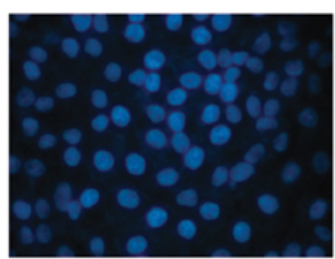

Control

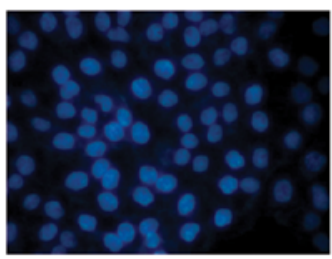

Bufalin (125 nM)

CAL 27/pcDNA cells

CAL 27/caAKT cells

B

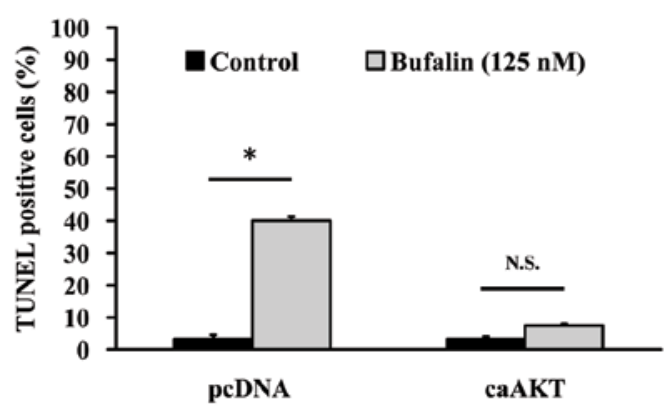

C

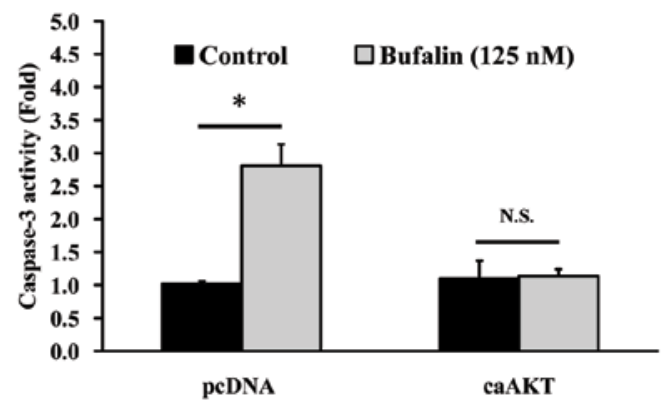

Figure 6. Bufalin-induced caspase-3-dependent apoptosis is mediated by AKT signaling in CAL 27/pcDNA or CAL 27/CA-AKT cells. The both types of cells were exposed to $125 \mathrm{nM}$ bufalin for $24 \mathrm{~h}$. (A) cells were stained with DAPI and analyzed under a fluorescence microscope followed by (B) TUNEL staining and flow cytometric analysis. The TUNEL positive cells were regarded as apoptotic. (C) Effect of bufalin on caspase-3 activity of CAL 27/pcDNA or CAL 27/CA-AKT cells. The results are mean $\pm \mathrm{SD}$ and representative of three independent experiments. " $\mathrm{p}<0.05$ versus the control group. 

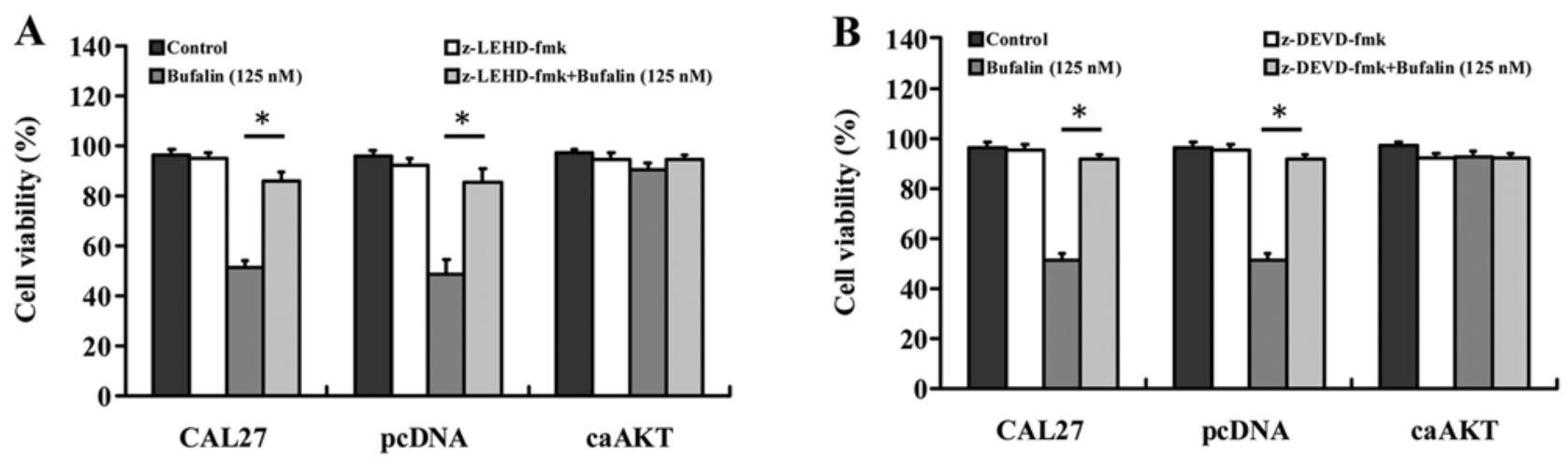

Figure 7. Bufalin induces intrinsic apoptosis via CA-AKT in CAL 27 cells. (A) Effect of the caspase-9 inhibitor (z-LEHD-fmk) on bufalin-induced apoptosis. CAL 27/pcDNA or CAL 27/CA-AKT cells were pre-incubated with or without the z-LEHD-fmk and then treated with $125 \mathrm{nM}$ of bufalin. (B) Effects of the caspase-3 inhibitor (z-DEVD-fmk) on bufalin-induced apoptosis as described in Materials and methods. The cellular viability was assessed by MTT assay. Data are mean \pm SD and representative of three independent experiments. "p $<0.05$ versus bufalin alone without the caspase- 9 or -3 inhibitor.

A

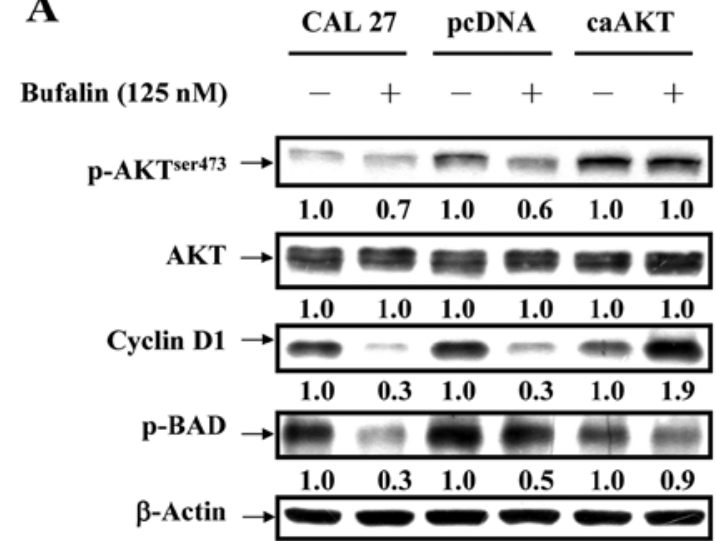

B

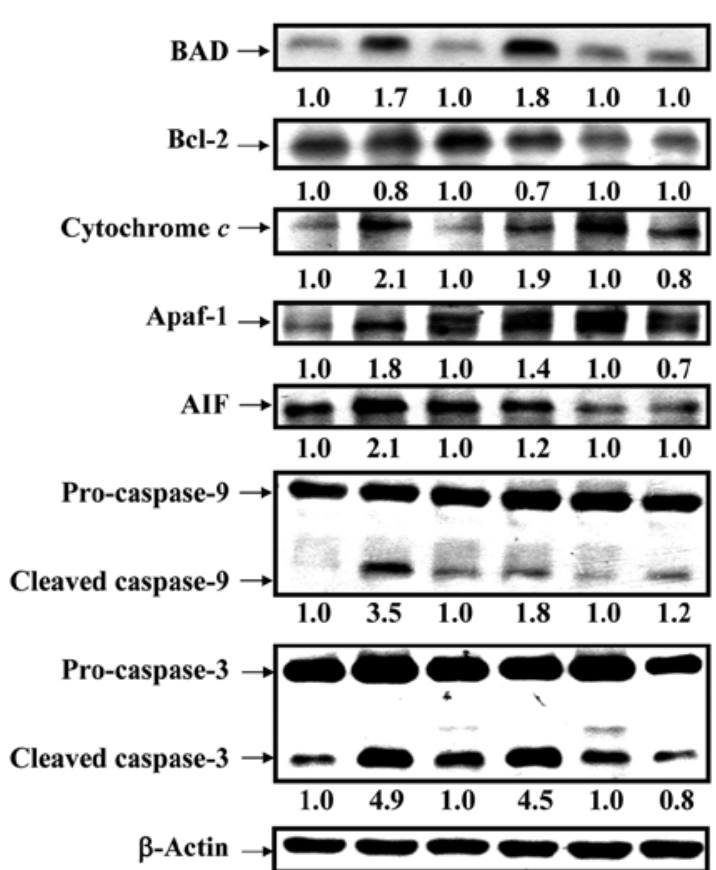

Figure 8. Bufalin alters the protein abundance-associated with mitochondria-dependent apoptotic signaling in CAL 27 cells. (A) Expressions of p-AKT ${ }^{\text {er473 }}$, AKT, cyclin D1 and p-BAD were analyzed by western blot analysis in CAL 27/pcDNA or CAL 27/CA-AKT cells after the treatment with $125 \mathrm{nM}$ of bufalin. (B) Expressions of BAD, Bcl-2, cytochrome $c$, Apaf-1 and AIF were measured after treatment. The cleavage of caspase-9, and caspase- 3 was determined by western blot analysis. Data are representative of three independent experiments. The blot was also probed with an anti- $\beta$-actin antibody to confirm equal loading of samples. Each band was quantified using ImageJ software.

caspase-9 inhibitor) blocked intracellular apoptotic proteases and attenuated bufalin-reduced viability and caused cell death in CAL 27 and CAL 27/pcDNA cells (Fig. 7A and B). Importantly, cells overexpressing CA-AKT had minimal effect on bufalin-induced cell death as can be seen in Fig. 7A and $\mathrm{B}$. These results suggest that bufalin-induced apoptosis in CAL 27 cells was associated with the mitochondria-mediated caspase-9 and caspase-3-dependent pathway.
Bufalin activates the mitochondrial apoptotic pathway involving the regulation of Bcl-2 family members through AKT signaling in CAL 27 cells. To confirm if AKT signal was overexpressed in CAL 27 cells, our data (Fig. 8A) showed that bufalin down-regulated level of AKT only when phosphorylated at threonine 308 but no alteration was observed in AKT expression in treated cells. Phosphorylated serine/ threonine protein kinase AKT has been reported to modu- 


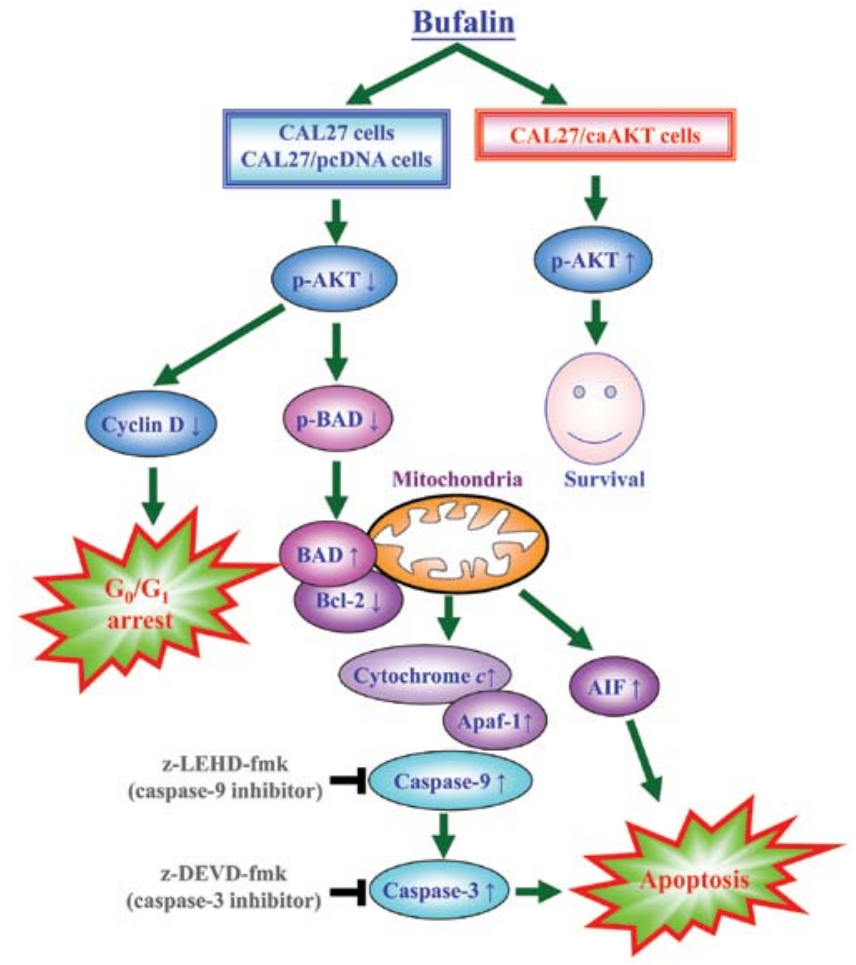

Figure 9. The proposed model of molecular mechanisms of bufalin-induced apoptosis in CAL 27 cells. Bufalin modulates p-AKT signal and upregulates BAD, downregulates Bcl-2 expression, activates mitochondria-mediated apoptotic pathway, which in turn causes the release of cytochrome $c$, the activation of caspase-9 and caspase-3, resulting in CAL 27 cell apoptosis.

late Bad phosphorylation at Ser136 (p-Bad) and cyclin D1 $(32,33)$. We found that the decreased expression of cyclin D1 and p-Bad occurred in CAL 27 and pcDNA/CAL 27 cells after exposure to bufalin. However, no dramatic effect on the levels of cyclin D1 and p-Bad was observed in caAKT/CAL 27 cells (Fig. 8A). Our results demonstrated the downstream of AKT signaling (cyclin D1 and p-BAD) was decreased in bufalintreated CAL 27 cells. Upon apoptotic signals, pro-apoptotic Bcl-2 members, such as BAD, was activated; in contrast, Bcl-2 can prevent this occurrence. The imbalance of expression of pro- and anti-apoptotic proteins is associated with the ultimate fate of cells $(33,34)$. To assess whether mitochondrial pathway is involved in bufalin-induced apoptosis, we evaluated the expression levels of BAD and Bcl-2 by western blot analysis. As shown in Fig. 8B, BAD protein levels increased, whereas Bcl-2 protein levels decreased after exposure of CAL 27 cells to $125 \mathrm{nM}$ bufalin for $24 \mathrm{~h}$. Thus, bufalin treatment increased the ratio of $\mathrm{BAD} / \mathrm{Bcl}-2$, which is in favor of the occurrence of apoptosis and leads to the release of cytochrome $c$ from mitochondria. In contrast, CAL 27 cells overexpressing constitutively active AKT (CA-AKT) had minimal effect on bufalin-induced cell death. Once released, cytochrome $c$ combines with apoptotic protease activating factor-1 (Apaf-1) and procaspase-9 to form the apoptosome in the presence of ATP, resulting in the activation of caspase- 9 and caspase-3 $(8,10)$. Next, we detected the expression of cytochrome $c$, Apaf- 1 and AIF in bufalin-treated cells. The expression of cytochrome $c$, Apaf-1 and AIF significantly increased after $24 \mathrm{~h}$ bufalin treatment (Fig. 8B). These results suggest that bufalin treatment-induced mitochondria-dependent apoptosis is mainly through AKT-regulated signaling (Fig. 9).

\section{Discussion}

Bufalin, a digitalis-like molecule from an animal source, functions as a $\mathrm{Na}^{+}-\mathrm{K}^{+}$-ATPase inhibitor and causes an increase in intracellular calcium in cancer cells (13-16). Just like several antitumor drugs including etoposide, adriamycin, and genistein, bufalin is also known as an inhibitor of topoisomerase II $(17,18)$. In addition, bufalin has been shown to induce apoptosis in a variety of human tumors, including colorectal carcinoma, melanoma, hepatoma, breast carcinoma, and gastric carcinoma (19-22). However, the molecular mechanisms responsible for the pro-apoptotic effects of bufalin in human oral cancer cells remain elusive.

Apoptotic characteristics include the appearance of the sub- $\mathrm{G}_{1}$ population and chromatin condensation and elevated pro-apoptotic protein expression levels, the cytochrome $c$ release and caspase cascade activation $(6,10,12)$. In the current study, we provided evidence that bufalin induced apoptosis in CAL 27 cells in a dose-dependent manner through cell cycle arrest at $G_{0} / G_{1}$ phase (Fig. 2), enhanced chromatin condensation by DAPI staining (Fig. 3A), up-regulation of pro-apoptotic proteins (Fig. 8A), the cytochrome $c$ (Figure 8B), and intrinsic caspase activation (Fig. 3C). Significant apoptotic death was observed using TUNEL assay, by bufalin in CAL 27 cells (Fig. 3B). Our results demonstrated that bufalin also serves as an apoptotic inducer in vitro.

Caspases participate in the execution of apoptosis $(8,11)$. There are two major caspase-dependent pathways. One is the death receptor induced caspase activation pathway, which results in caspase- 8 activation. The other is the mitochondrial apoptotic pathway, dependent on the release of cytochrome $c$ from mitochondria to the cytosol. Released cytochrome $c$ binds with Apaf-1 and activates caspase-9, which then activates caspase-3 $(6,12)$. Our study revealed that bufalin failed to activate the death receptor-mediated caspase-8 pathway in CAL 27 cells (data not shown). In contrast, we observed that the process of bufalininduced apoptosis is involved the activation of caspase- 9 and -3 , and that the treatment with specific inhibitors of caspase- 9 and -3 significantly prevented the bufalin-induced cell apoptotic effects (Figs. 7 and 8B). Thus, our results demonstrated that the bufalin-induced apoptosis is carried out through the mitochondria-dependent response. Additionally, cytochrome $c$-mediated apoptosis is controlled prominently by the members of $\mathrm{Bcl}-2$ family. BAD and Bcl-2 have been identified as major regulators. BAD possesses proapoptotic ability, while Bcl-2 blocks apoptosis. Therefore, the balance between the levels of Bcl-2 and BAD is important in determining cell survival or death $(8,10)$. Our finding shown in the present study demonstrated that bufalin-treatment increased the ratio of $\mathrm{BAD} / \mathrm{Bcl}-2$, suggesting that the increase of the ratio of $\mathrm{BAD} / \mathrm{Bcl}-2$ might be the key factor of bufalin-induced apoptosis (Fig. 8A).

Next, we investigated whether the possible mechanism of bufalin-induced apoptosis is through activation of the AKT signaling pathway in CAL 27 cells (Figs. 4-8). AKT is a serinethreonine kinase and regulates cancer cell progression. It has been demonstrated that AKT is over-activated or over-expressed in many human malignancies $(5,25)$. Understanding the control of the Akt signaling pathway is potentially important for developing therapeutic inhibitors. CAL 27 cells overexpressing constitutively active AKT (CA-AKT) is used for evaluating 
bufalin-induced cell death. Data demonstrated that CA-AKT diminished bufalin-induced cell apoptosis. Hence, our study is the first report regarding AKT signaling contributed to bufalin-triggered mitochondrial apoptotic death in CAL 27 cells in vitro.

In summary, our results provided further insight into bufalin-induced apoptosis and deepen our knowledge on the mechanisms of anticancer activity of bufalin in CAL 27 cells. Bufalin causes cell cycle arrest at the $G_{0} / G_{1}$ phase. The bufalin-induced apoptosis is dependent on the mitochondria-mediated caspase activation and involvement of the regulation of Bcl-2 and BAD (Fig. 8). An exciting finding in this study is that constitutively active AKT had no significant effect on bufalin-induced apoptosis. These data provide a hint toward clarification of the mechanisms of bufalin-induced apoptosis, but it might be a long way before unveiling the complete mechanisms underlying bufalin-induced apoptosis in tumor cells. Moreover, other signaling components such as FOXO3a, p2 $7^{\mathrm{KIP} 1}$, and c-Myc might be also involved in bufalin-induced apoptosis $(35,36)$. We believe that bufalin has important antitumor properties and is a promising chemotherapeutic agent for the treatment of human oral cancer in the future. Continued examination of AKT and other signaling pathways will be important in further delineating the cell death mechanisms. Work ongoing in our laboratory is addressing these issues.

\section{Acknowledgements}

This study was supported in part by a research grant from the National Science Council of the Republic of China (NSC-101-2313-B-039-008) awarded to Dr Jai-Sing Yang and partly by the grant from Cancer Research Center of Excellence, China Medical University Hospital, Taiwan Department of Health, (DOH101-TD-C-111-005) awarded to Dr Sheng-Chu Kuo.

\section{References}

1. Chien MH, Ying TH, Hsieh YS, et al: Dioscorea nipponica Makino inhibits migration and invasion of human oral cancer HSC-3 cells by transcriptional inhibition of matrix metalloproteinase-2 through modulation of CREB and AP-1 activity. Food Chem Toxicol 50: 558-566, 2012.

2. Spiro RH, Alfonso AE, Farr HW and Strong EW: Cervical node metastasis from epidermoid carcinoma of the oral cavity and oropharynx. A critical assessment of current staging. Am J Surg 128: 562-567, 1974.

3. Liu SY, Lu CL, Chiou CT, et al: Surgical outcomes and prognostic factors of oral cancer associated with betel quid chewing and tobacco smoking in Taiwan. Oral Oncol 46: 276-282, 2010.

4. Funk GF, Karnell LH, Robinson RA, Zhen WK, Trask DK and Hoffman HT: Presentation, treatment, and outcome of oral cavity cancer: a National Cancer Data Base report. Head Neck 24: 165-180, 2002.

5. Chung JG, Yang JS, Huang LJ, et al: Proteomic approach to studying the cytotoxicity of YC-1 on U937 leukemia cells and antileukemia activity in orthotopic model of leukemia mice. Proteomics 7: 3305-3317, 2007.

6. Sanjiv K, Su TL, Suman S, et al: The novel DNA alkylating agent BO-1090 suppresses the growth of human oral cavity cancer in xenografted and orthotopic mouse models. Int $\mathbf{J}$ Cancer 130: 1440-1450, 2012.

7. Sun Q, Sakaida T, Yue W, Gollin SM and Yu J: Chemosensitization of head and neck cancer cells by PUMA. Mol Cancer Ther 6: 3180-3188, 2007.
8. Kroemer G, Galluzzi L and Brenner C: Mitochondrial membrane permeabilization in cell death. Physiol Rev 87: 99-163, 2007.

9. Lai E, Teodoro T and Volchuk A: Endoplasmic reticulum stress: Signaling the unfolded protein response. Physiology (Bethesda) 22: 193-201, 2007.

10. Orrenius S: Reactive oxygen species in mitochondria-mediated cell death. Drug Metab Rev 39: 443-455, 2007.

11. Lu CC, Yang JS, Chiang JH, et al: Novel quinazolinone MJ-29 triggers endoplasmic reticulum stress and intrinsic apoptosis in murine leukemia WEHI-3 cells and inhibits leukemic mice. PLoS One 7: e36831, 2012.

12. Lavrik IN, Golks A and Krammer PH: Caspases: Pharmacological manipulation of cell death. J Clin Invest 115: 2665-2672, 2005.

13. Takai N, Kira N, Ishii T, et al: Bufalin, a traditional oriental medicine, induces apoptosis in human cancer cells. Asian Pac J Cancer Prev 13: 399-402, 2012.

14. Krenn L and Kopp B: Bufadienolides from animal and plant sources. Phytochemistry 48: 1-29, 1998.

15. Yu CH, Kan SF, Pu HF, Jea Chien E and Wang PS: Apoptotic signaling in bufalin- and cinobufagin-treated androgen-dependent and -independent human prostate cancer cells. Cancer Sci 99: 2467-2476, 2008.

16. Bagrov AY, Roukoyatkina NI, Fedorova OV, Pinaev AG and Ukhanova MV: Digitalis-like and vasoconstrictor effects of endogenous digoxin-like factor(s) from the venom of Bufo marinus toad. Eur J Pharmacol 234: 165-172, 1993.

17. Bandele OJ and Osheroff N: The efficacy of topoisomerase II-targeted anticancer agents reflects the persistence of drug-induced cleavage complexes in cells. Biochemistry 47: 11900-11908, 2008.

18. Baldwin EL and Osheroff N: Etoposide, topoisomerase II and cancer. Curr Med Chem Anticancer Agents 5: 363-372, 2005.

19. Huang WW, Yang JS, Pai SJ, et al: Bufalin induces $\mathrm{G}(0) / \mathrm{G}(1)$ phase arrest through inhibiting the levels of cyclin D, cyclin $\mathrm{E}$, CDK2 and CDK4, and triggers apoptosis via mitochondrial signaling pathway in T24 human bladder cancer cells. Mutat Res 732: 26-33, 2012.

20. Masuda Y, Kawazoe N, Nakajo S, Yoshida T, Kuroiwa Y and Nakaya K: Bufalin induces apoptosis and influences the expression of apoptosis-related genes in human leukemia cells. Leuk Res 19: 549-556, 1995.

21. Qi F, Inagaki Y, Gao B, et al: Bufalin and cinobufagin induce apoptosis of human hepatocellular carcinoma cells via Fas- and mitochondria-mediated pathways. Cancer Sci 102: 951-958, 2011.

22. Han KQ, Huang G, Gu W, Su YH, Huang XQ and Ling CQ: Anti-tumor activities and apoptosis-regulated mechanisms of bufalin on the orthotopic transplantation tumor model of human hepatocellular carcinoma in nude mice. World J Gastroenterol 13: 3374-3379, 2007.

23. Aoki M, Batista O, Bellacosa A, Tsichlis P and Vogt PK: The akt kinase: Molecular determinants of oncogenicity. Proc Natl Acad Sci USA 95: 14950-14955, 1998.

24. Brognard J, Clark AS, Ni Y and Dennis PA: Akt/protein kinase $\mathrm{B}$ is constitutively active in non-small cell lung cancer cells and promotes cellular survival and resistance to chemotherapy and radiation. Cancer Res 61: 3986-3997, 2001.

25. Lu CC, Yang JS, Huang AC, et al: Chrysophanol induces necrosis through the production of ROS and alteration of ATP levels in J5 human liver cancer cells. Mol Nutr Food Res 54: 967-976, 2010

26. Wu PP, Liu KC, Huang WW, et al: Triptolide induces apoptosis in human adrenal cancer NCI-H295 cells through a mitochondrial-dependent pathway. Oncol Rep 25: 551-557, 2011.

27. Yang JS, Hour MJ, Huang WW, Lin KL, Kuo SC and Chung JG: MJ-29 inhibits tubulin polymerization, induces mitotic arrest, and triggers apoptosis via cyclin-dependent kinase 1-mediated BCL-2 phosphorylation in human leukemia U937 cells. J Pharmacol Exp Ther 334: 477-488, 2010.

28. Chiang JH, Yang JS, Ma CY, et al: Danthron, an anthraquinone derivative, induces DNA damage and caspase cascades-mediated apoptosis in SNU-1 human gastric cancer cells through mitochondrial permeability transition pores and Bax-triggered pathways. Chem Res Toxicol 24: 20-29, 2011.

29. Wu SH, Hang LW, Yang JS, et al: Curcumin induces apoptosis in human non-small cell lung cancer NCI-H460 cells through ER stress and caspase cascade- and mitochondria-dependent pathways. Anticancer Res 30: 2125-2133, 2010. 
30. Ji BC, Hsu WH, Yang JS, et al: Gallic acid induces apoptosis via caspase-3 and mitochondrion-dependent pathways in vitro and suppresses lung xenograft tumor growth in vivo. J Agric Food Chem 57: 7596-7604, 2009.

31. Huang WW, Chiu YJ, Fan MJ, et al: Kaempferol induced apoptosis via endoplasmic reticulum stress and mitochondriadependent pathway in human osteosarcoma U-2 OS cells. Mol Nutr Food Res 54: 1585-1595, 2010.

32. Weng LP, Brown JL and Eng C: PTEN coordinates G(1) arrest by down-regulating cyclin d1 via its protein phosphatase activity and up-regulating p 27 via its lipid phosphatase activity in a breast cancer model. Hum Mol Genet 10: 599-604, 2001.

33. Kuo CT, Hsu MJ, Chen BC, et al: Denbinobin induces apoptosis in human lung adenocarcinoma cells via akt inactivation, bad activation, and mitochondrial dysfunction. Toxicol Lett 177: 48-58, 2008.
34. Qin J, Xie LP, Zheng XY, et al: A component of green tea, (-)-epigallocatechin-3-gallate, promotes apoptosis in T24 human bladder cancer cells via modulation of the PI3K/Akt pathway and Bcl-2 family proteins. Biochem Biophys Res Commun 354: 852-857, 2007.

35. Li D, Qu X, Hou K, et al: PI3K/Akt is involved in bufalininduced apoptosis in gastric cancer cells. Anticancer Drugs 20: 59-64, 2009.

36. Kawazoe N, Watabe M, Masuda Y, Nakajo S and Nakaya K: Tiam1 is involved in the regulation of bufalin-induced apoptosis in human leukemia cells. Oncogene 18: 2413-2421, 1999. 